\title{
Selectivity index and separation efficiency prediction in industrial magnetic separation process using a hybrid neural genetic algorithm
}

\author{
Usef Paledi ${ }^{1}$ Ebrahim Allahkarami ${ }^{2} \cdot$ Bahram Rezai $^{2} \cdot$ Mohammad Reza Aslani $^{2}$
}

Received: 5 October 2020 / Accepted: 10 February 2021 / Published online: 19 February 2021

(C) The Author(s) 2021 OPEN

\begin{abstract}
It is essential to know the process efficiency in the industrial magnetic separation process under different operating conditions because it is required to control the process parameters to optimize the process efficiency. To our knowledge, there is no information about using artificial intelligence for modeling the magnetic separation process. Hence, finding a robust and more accurate estimation method for predicting the separation efficiency and selectivity index is still necessary. In this regard, a feed-forward neural network was developed to predict the separation efficiency and selectivity index. This model was trained to present a predictive model based on the percentage of iron, iron oxide and sulfur in mill feed and cobber feed, $80 \%$ passing size in mill feed and cobber feed and plant capacity. Therefore, this work aims to develop an intelligent technique based on an artificial neural network and a hybrid neural-genetic algorithm for modeling the concentration process. Results indicated that the values of mean square error and coefficient of determination for the testing phase were obtained 0.635 and 0.86 for selectivity index and of 4.646 and 0.84 for separation efficiency, respectively. In order to improve the performance of neural network, genetic algorithm was used to optimize the weights and biases of neural network. The results of modeling with GA-ANN technique indicated that the mean square error and coefficient of determination for the testing phase were achieved by 0.276 and 0.95 for selectivity index and of 1.782 and 0.92 for separation efficiency, respectively. The other statistical criteria for the GA-ANN model were better than those of the ANN model.
\end{abstract}

Keywords Magnetic separation process · Modeling · Prediction · Artificial neural network · Genetic algorithm

\section{Introduction}

Due to the increasing demand for iron, the management of these resources is an important subject of recent researches, which has attracted researchers to apply a robust reference tracking control strategy for managing these resources. The use of modeling and simulation is a well-accepted technique in many processes, especially in mineral processing. The prediction of efficiency is an important parameter for processes applied in mineral processing. In the industrial magnetic separation process, the online prediction of separation efficiency is costly and time-consuming. To overcome these difficulties, the use of statistical and artificial intelligence techniques for monitoring purposes has been developed. Artificial intelligence methods were found to be more efficient than statistical methods for the prediction of process efficiency.

Artificial intelligence techniques, such as support vector machine (SVM) [1], random forest (RF) [2], adaptive neural-based fuzzy inference system (ANFIS) [3], radial basis function neural network (RBFNN) [4] and artificial neural network (ANN) [5] have been efficiently used in the modeling and simulation of processes. Artificial neural networks employ very powerful computational techniques

Ebrahim Allahkarami, Ebrahim.allahkarami@aut.ac.ir| ${ }^{1}$ Mining Engineering Group, Shahid Bahonar University of Kerman, Kerman, Iran. ${ }^{2}$ Department of Mining Engineering, Amirkabir University of Technology, Tehran, Iran. 
for modeling complex nonlinear relationships. They can be applied to solve and model many complicated processes due to their acceptable accuracy, ease of modeling, robustness, simplicity and nonlinearity.

Artificial neural networks estimate complex nonlinear associates existing between input and output variables to an arbitrary degree of accuracy. Recently, neural networks have been applied as a powerful modeling tool in different processes such as adsorption [6], flotation [7], liquid-liquid extraction [8] and many other fields of modeling problems $[9,10]$. They could be successfully used in regression and interpolating problems. For example, Allahkarami et al. [5] used an artificial neural network to estimate copper grade and recovery based on operational parameters. Jahedsaravani et al. [3] have applied intelligent techniques (i.e., neural network and adaptive neuro-fuzzy method) and statistical approach (i.e., nonlinear regression) for modeling the metallurgical parameters in the batch flotation process. Their results indicated that intelligent techniques outperform statistical approaches. Medi et al. [10] have used artificial neural network models with a novel k-fold crossvalidation technique to optimize minimum film thickness, maximum transparency and maximum conductivity. Their results showed that the developed model could predict the film characteristics with a reasonable error. Tripathy et al. [11] applied an artificial neural network to predict the performance of induced roll high-intensity magnetic separator. Four variables including applied current, rotor speed, feed rate and splitter position were used to develop a network for predicting the performance of the separator. All data were normalized in the range of -1 and +1 . Mean square error was used to evaluate the performance of the developed model for the determination of the optimal structure. They found that the proposed model provides realistic predictive performance $\left(R^{2}>0.95\right)$. Some scholars studied the modeling of metallurgical parameters in the flotation process through statistical and intelligent techniques. Their results indicated that intelligent techniques (i.e., neural network and adaptive neuro-fuzzy) are more efficient tools than statistical approaches (i.e., nonlinear regression) [3]. In other work, data mining, neural network and time series analysis were applied to evaluate and model the copper concentrator. They used data mining for selecting the most effective variables on the metallurgical performance of the process. Modeling and predicting the future trend of copper concentrator were conducted by ANN and time series analysis, respectively [12]. Tohry et al. [2] used Pearson correlation and random forest methods for optimization and variable assessments for SLon ${ }^{\oplus}$ operating variables to evaluate their effects on separation efficiency. Hu et al. [13] combined a genetic algorithm approach with pulp and froth modeling in different flotation cells to optimize the flotation circuit layout for
3-8 cells (Hu et al. 2013). However, various statistical and intelligent techniques have been applied to simulate the separation process in mineral processing industries. Some of them are unsuitable for deep learning, relatively slow and relatively complex for design. Using random initial weights for neural network training may lead to trapping into the local minima and slow converging. As a result, the NN has often been unable to find a desirable solution. So, in this study, genetic algorithm was used to optimize weights and biases of neural networks to improve the performance of ANN.

To our knowledge, there is no information about using artificial intelligence for modeling the magnetic separation process. Hence, finding a robust and more accurate estimation method for predicting the separation efficiency and selectivity index is still necessary. Therefore, this work aims to develop an intelligent technique based on artificial neural network and hybrid neural genetic algorithm for modeling the concentration process. To evaluate the performance of the proposed model, the statistical criteria, including the coefficient determination $\left(R^{2}\right)$, mean square error (MSE), median absolute deviation (MAD) and mean absolute percentage error (MAPE), are used.

\section{Description of industrial magnetic separation process}

The Golgohar ore body is located in southeast Iran, which contains half billion tons of ore having about $42 \%$ iron. Golgohar Mining and Industrial Company operates six separated ore bodies of Golgohar. It produces 10.8 million tons of iron ore concentrate, 157 thousand tons of fine iron ore and 5.3 million tons of iron ore pellets. Golgohar Mining and Industrial Company has four mineral processing plants, including concentration plant, hematite recovery, pelletizing and Polycom iron ore concentrate plant for magnetic separation of iron ore. Flow sheet of Polycom iron ore concentrate plant is shown in Fig. 1. It consists of primary crushing, cone crushing, screening, milling, magnetic separation, thickening and filtering.

In this study, the percentage of $\mathrm{Fe}, \mathrm{FeO}$ and $\mathrm{S}$ in mill feed and cobber feed, $80 \%$ passing size in mill feed and cobber feed and plant input were considered as the inputs to the network. Table 1 gives the summary statistics for each input variable.

The percentage of $\mathrm{FeO}$ in the final concentrate fluctuates between 23 and $26 \%$, depending on operating conditions and ore type. The presence of sulfur in iron concentrate affects the price of iron ore concentrate because sulfur in steel decreases its quality. Furthermore, sulfur in iron concentrates causes environmental problems, especially sulfur dioxide emissions during the smelting 


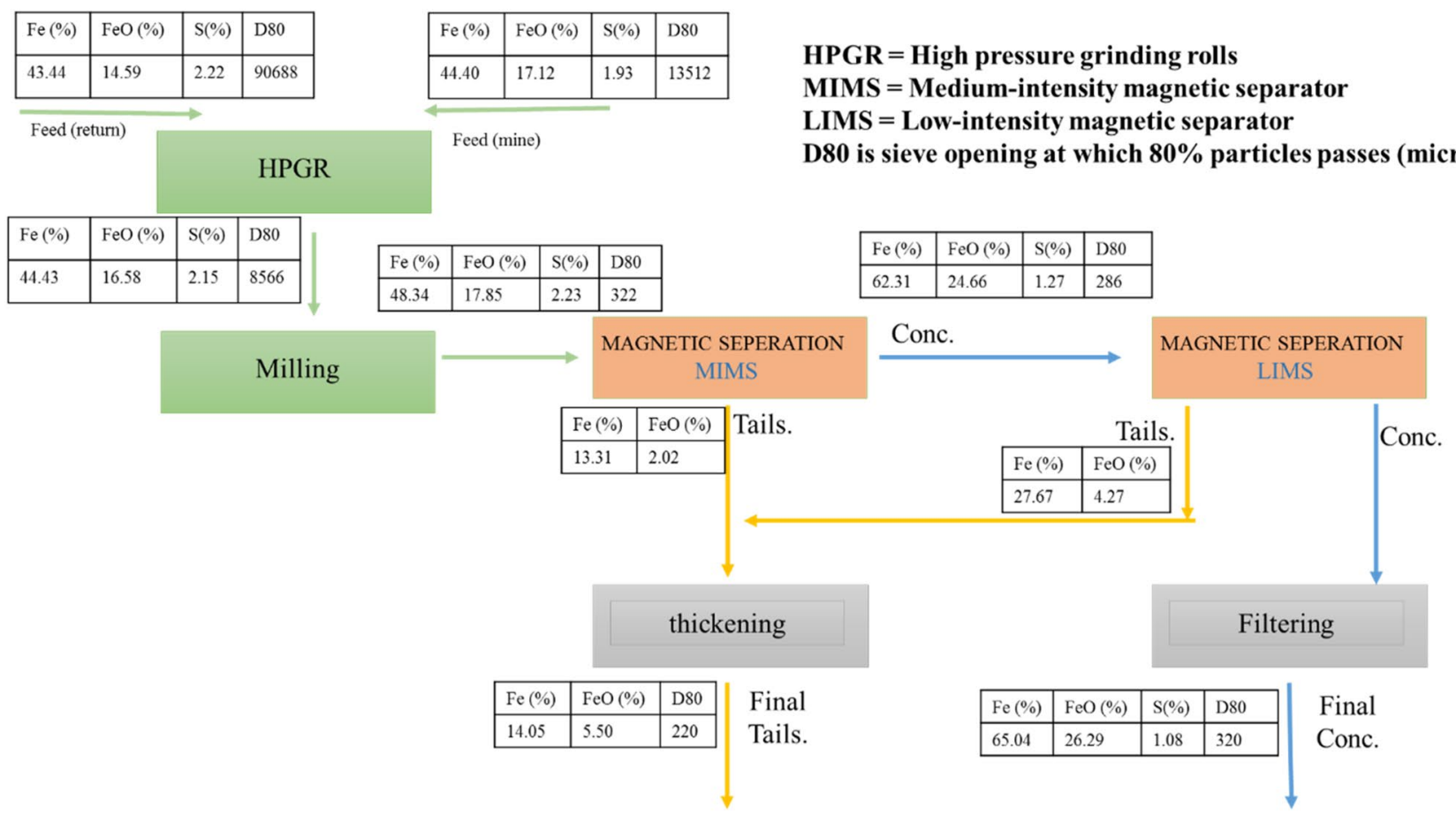

Fig. 1 The simplified flow sheet of Polycom iron ore concentrate circuit

Table 1 The summary statistics for input variables

\begin{tabular}{llllll}
\hline Variable & Index & Min & Max & Mean & $\begin{array}{l}\text { Standard } \\
\text { deviation }\end{array}$ \\
\hline Fe grade in feed (\%) & & & & & 3.02 \\
FeO grade in feed (\%) & Fe-F & 35.34 & 52.27 & 44.90 & 3.21 \\
S grade in feed (\%) & Feo-F & 9.17 & 55.36 & 15.73 & 0.49 \\
Fe grade in cobber (\%) & S-F & 0.68 & 3.92 & 2.00 & 3.34 \\
FeO grade in cobber (\%) & Fe-C & 21.22 & 55.91 & 48.61 & 1.48 \\
S grade in cobber (\%) & Feo-C & 11.70 & 20.96 & 16.88 & 0.366 \\
Plant input (ton) & S-C & 1.142 & 3.532 & 2.003 & 6,909 \\
80\% passing size in mill feed $\left(\mathrm{d}_{80}\right)$ & Inp & 968 & 100,634 & 8,755 & 1,836 \\
$80 \%$ passing size in cobber feed $\left(\mathrm{d}_{80}\right)$ & Pas-M & 350 & 12,776 & 7,352 & 36.850 \\
\hline
\end{tabular}

or pelletizing processes. So, the separation of $\mathrm{S}$ from iron $(\mathrm{Fe})$ is important. Grade and recovery are the most widely accepted indices to evaluate a process. There are different indices for evaluating mineral processing processes, such as separation efficiency, selectivity index, operation efficiency and efficiency ratio [14]. Separation efficiency index is the combination of grade and recovery, and selectivity index is a measure for evaluating two-product separation. In this study, two goals of this separation process are (1) to separate sulfur from iron (selectivity index is a suitable index for the evaluation of this separation process) and (2) to measure the recovery of $\mathrm{FeO}$ in concentrate regarding the recovery of sulfur in tailing (separation efficiency is a suitable index for the evaluation of this separation process). Therefore, two indices named separation efficiency (SE) and selectivity index (SI) were used. These indices are calculated by Equations (1) and (2) [15, 16]:

$S E=R_{m}-R_{g}$

$S I=\sqrt{\frac{R_{a} \times R_{b}}{\left(100-R_{a}\right) \times\left(100-R_{b}\right)}}$

where $R_{m}$ and $R_{g}$ are the recoveries of valuable and gangue minerals, respectively; $R_{a}$ and $R_{b}$ are the recovery of iron $(\mathrm{Fe})$ in concentrate and the recovery of sulfur $(\mathrm{S})$ in tailing fraction. So, variables of the separation efficiency and 
selectivity index were used as the network outputs. Table 2 presents the summary statistics for each output variable.

In this research, ANN and GA-ANN models were used and compared with each other. All calculations were carried out using Matlab 2017b for windows, which were run on a personal computer (Pentium V 2.3 GHz). All 193 datasets were randomly selected to train and test the developed models. So, $70 \%$ of all data (135) was randomly considered to train the model, whereas $30 \%$ of them (58) was selected to test the network. To facilitate modeling and speed training, all data before using in models were normalized in the range of -1 and 1 based on the following equation:

$X_{N}=2 \frac{X-X_{\min }}{X_{\max }-X_{\min }}-1$

where $X_{N}$ is the normalized value of each parameter; $X$, $X_{\max }$ and $X_{\min }$ are original, maximum and minimum values of the parameters, respectively.

\section{Model description}

\subsection{Artificial neural network}

Recently, soft computing methods have been successfully used in mineral processing systems $[17,18]$. They are used for solving very complex engineering problems. The most commonly used method in soft computing methods is artificial neural network (ANN) [19]. It consists of a large number of neurons in several layers (input, hidden and output layers). In fact, these neurons are information processing units that have been used for designing the artificial neural network. They are connected by weighted links and bias. The output of each neuron is transferred to the next layer as an input. Finally, the nonlinear basis function set is used to calculate the outputs of ANN, using Eq. 4.

$y_{j}=\sum_{i=1}^{n} f\left(w_{i j} x_{i}\right)+b_{j}$

where $x_{i}$ and $y_{i}$ are input and output variables, respectively; $w_{i j}$ is the synaptic weight of neuron $k$; $f($.$) is the acti-$ vation function and $b_{k}$ is the bias.

Table 2 The summary statistics for output variables

\begin{tabular}{llrlrl}
\hline Variable & Index & Min & Max & Mean & $\begin{array}{l}\text { Standard } \\
\text { deviation }\end{array}$ \\
\hline SI Fe-S & SI & 3.355 & 27.547 & 7.812 & 1.948 \\
$\begin{array}{l}\text { Separation } \\
\text { efficiency } \\
(\%)\end{array}$ & SE & 40.277 & 81.638 & 57.796 & 5.650 \\
\hline
\end{tabular}

\subsection{Hybrid neural genetic algorithm}

The backpropagation (BP) algorithm is a common method of neural network that adjusts weights communication and biases according to gradient descent to minimize the error between predicted and target value. BP has some defects, including slow converging and trapping into the local minima [20]. To overcome these defects, the genetic algorithm (GA) can be applied to optimize the initial weights and biases.

The genetic algorithm as a search heuristic algorithm is a suitable method for the optimization process [21]. This method has been successfully used in mineral processing systems for parameter optimization and circuit design [22-24]. The operators in GA are briefly explained in the following:

- Generation of an initial population

The variables to be optimized are considered as a chromosome. In fact, each chromosome has $\mathrm{M}$ variables named Gen. So, GA is meeting a problem of $\mathrm{N}$-dimensional optimization. The GA starts optimization with some chromosomes, which is called a population. Population size means that how many chromosomes are in one generation of population. If this size is small, GA has a little search space to be explored; if the size of the population is large, the computational load will become high and it does not make solving the problem faster. Therefore, in this research, the population size was considered 100.

- Fitness evaluation

In each generation, a fitting function was applied to evaluate all the chromosomes. Then, the chromosomes with the best value of fitness function are chosen.

- Reproduction of a new population

After selecting the best chromosomes, they are considered as parents. Some selection operators include roulette wheel, tournament, ranking, uniform selection operators, etc. Also, some crossover operators include single point, two points, heuristic, arithmetic, scattered crossover, etc. Then, new chromosomes are generated by crossover and mutation operators. A crossover is used to vary the programming of a chromosome from one generation to the next. It is being applied over two individuals chosen by selection operators to produce offsprings from them. The crossover probability of this research was 0.5 . It means that offsprings are made from parts of parent's chromosome. The mutation operator randomly changes the values of each element of chromosomes based on the mutation probability. Mutation probability means that how often parts of chromosome will be muted. The mutation rate is in the range of 0 and 1 . In this research, the mutation rate was 
considered 0.03 . There are different mutation operators in real-coded GA, such as uniform, non-uniform, boundary mutation and multi-non-uniform [25]. After applying these operators, the offsprings are generated and their fitness values are calculated.

Table 3 GA parameters used in this research

\begin{tabular}{ll}
\hline Parameters & Value \\
\hline Crossover probability & 0.5 \\
Mutation probability & 0.03 \\
Population size & 100 \\
Number of generation & 100 \\
\hline
\end{tabular}

- Termination criterion

The previous step, reproduction, is continued until a stopping condition is achieved. Termination criterion can be the number of generations. In this study, the number of generations was considered 100 generations. In this research, GA is used for the optimization of initial weights and biases of NN to improve the performance of it for estimation of process outputs. The GA parameters for this study are summarized in Table 3.

As mentioned in the previous section, GA as a powerful method in evolutionary computation research can be applied for solving a variety of optimization problems. In this study, GA is applied to find the optimal connection weights and biases of ANN. The flowchart of combining GA and ANN is shown in Fig. 2 (right).

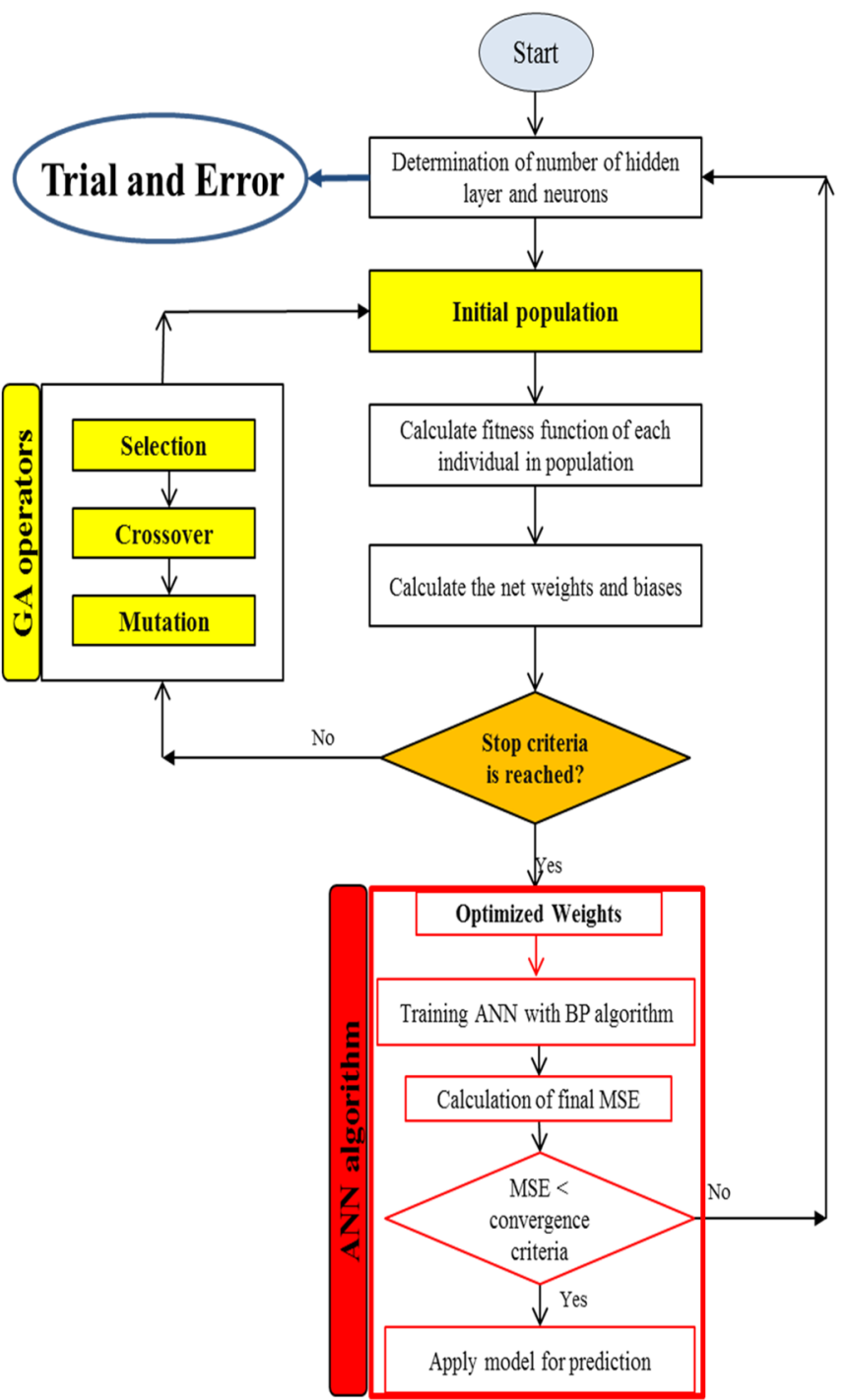

Fig. 2 Flowchart of (Left) ANN model and (Right) GA-ANN model 
As shown in Fig. 2 (left), a feed-forward neural network was used for modeling and prediction. The term backpropagation refers to the process by which derivatives of the network error, with respect to network weights and biases, can be computed. This method is based on the backpropagation error algorithm, which is an iterative supervised learning technique. During each iteration, the error signal travels backward through the network, starting at the output neurons and ending at the input synapses. The neural network learns the relations contained in between the input and the output variables and correlates the variables by the optimal weights that minimize the differences between the estimated and observed output values. The performance criteria were MSE, that is the average squared error between the predicted and the observed outputs. Using this algorithm, the main ANN structure was constructed and trained. Training of backpropagation will be done with random initial weights. One of the important applications of genetic algorithm (GA) is applying this technique to learn the weights of NN. So, after optimization of weights and biases, they were considered for initial weights of NN. After that, the backpropagation learning algorithm was used to train NN. For two models, the error between the predicted and the observed values for each iteration is propagated backward from the output layer toward the input layer through the hidden layers. This work continued until the predicted and the target values are in good agreement (the convergence criteria are met).

\subsection{Model evaluation criteria}

To evaluate the performance of the developed model for estimation of the separation efficiency and selectivity index of magnetic separation process under different operating conditions, the following statistical measures are employed:

$$
\begin{aligned}
& R^{2}=1-\frac{\sum_{i=1}^{n}\left(y_{i}-x_{i}\right)^{2}}{\sum_{i=1}^{n}\left(y_{i}-x_{i}\right)^{2}+\sum_{i=1}^{n}\left(x_{i}-\bar{x}\right)^{2}} \\
& M S E=\frac{1}{n} \sum_{i=1}^{n}\left(x_{i}-y_{i}\right)^{2} \\
& M A D=\frac{1}{n} \sum_{i=1}^{n}\left|x_{i}-y_{i}\right| \\
& \text { MAPE }=\frac{1}{n} \sum_{i=1}^{n}\left|\frac{x_{i}-y_{i}}{x_{i}}\right| * 100
\end{aligned}
$$

where $R^{2}$, MSE, MAD and MAPE are the coefficient of determination, mean square error, median absolute deviation and mean absolute percentage error, respectively; $\mathrm{n}$ is the number of samples; $\bar{x}$ is the mean value of target values; $x_{i}$ and $y_{i}$ are the target and predicted values, respectively.

\section{Results and discussion}

The best structure of ANN can be determined by changing the function type of training and transfer function, the hidden layer size and the number of neurons in each layer. In fact, the appropriate structure of ANN is found by a trial and error method [26]. In this study, several networks were generated, trained and tested. The ANN model has been developed by considering two hidden layers in the MLP configuration and training using the backpropagation algorithm. During each iteration, the error signal travels backward through the network, starting at the output neurons and ending at the input synapses. The NN trains the relation between the input and the output parameters and correlates the parameters using the connection weights and biases that minimizes the differences between the observed and the predicted values. Due to the convergence speed and the performance of the network to find a better solution, a learning rule was selected by the Levenberg-Marquardt training method. Without momentum, a network may be trapped in a local minimum. Therefore, optimum values of 0.1 and 0.9 were selected for learning rate and momentum, respectively. Using this algorithm, the main ANN structure was constructed and trained. Finally, a feed-forward neural network with the arrangement of 9:18:16:2 was used for modeling. The parameters of developed model are given in Table 4. The coefficient of determination $\left(R^{2}\right)$ in the training and the testing stages are shown in Fig. 3. The statistical

Table 4 The basic architecture and the training parameters of the ANN

\begin{tabular}{ll}
\hline Parameter & ANN \\
\hline Number of layers & 4 \\
Number of neurons in the input layer & 9 \\
Number of neurons in the first hidden layer & 18 \\
Number of neurons in the second hidden layer & 16 \\
Number of neurons in the output layer & 2 \\
Transfer function of the first hidden layer & Tan sigmoid \\
Transfer function of the second hidden layer & Tan sigmoid \\
Transfer function of the output layer & Linear \\
Number of epochs & 1000 \\
Learning rate & 0.1 \\
Momentum & 0.9 \\
\hline
\end{tabular}



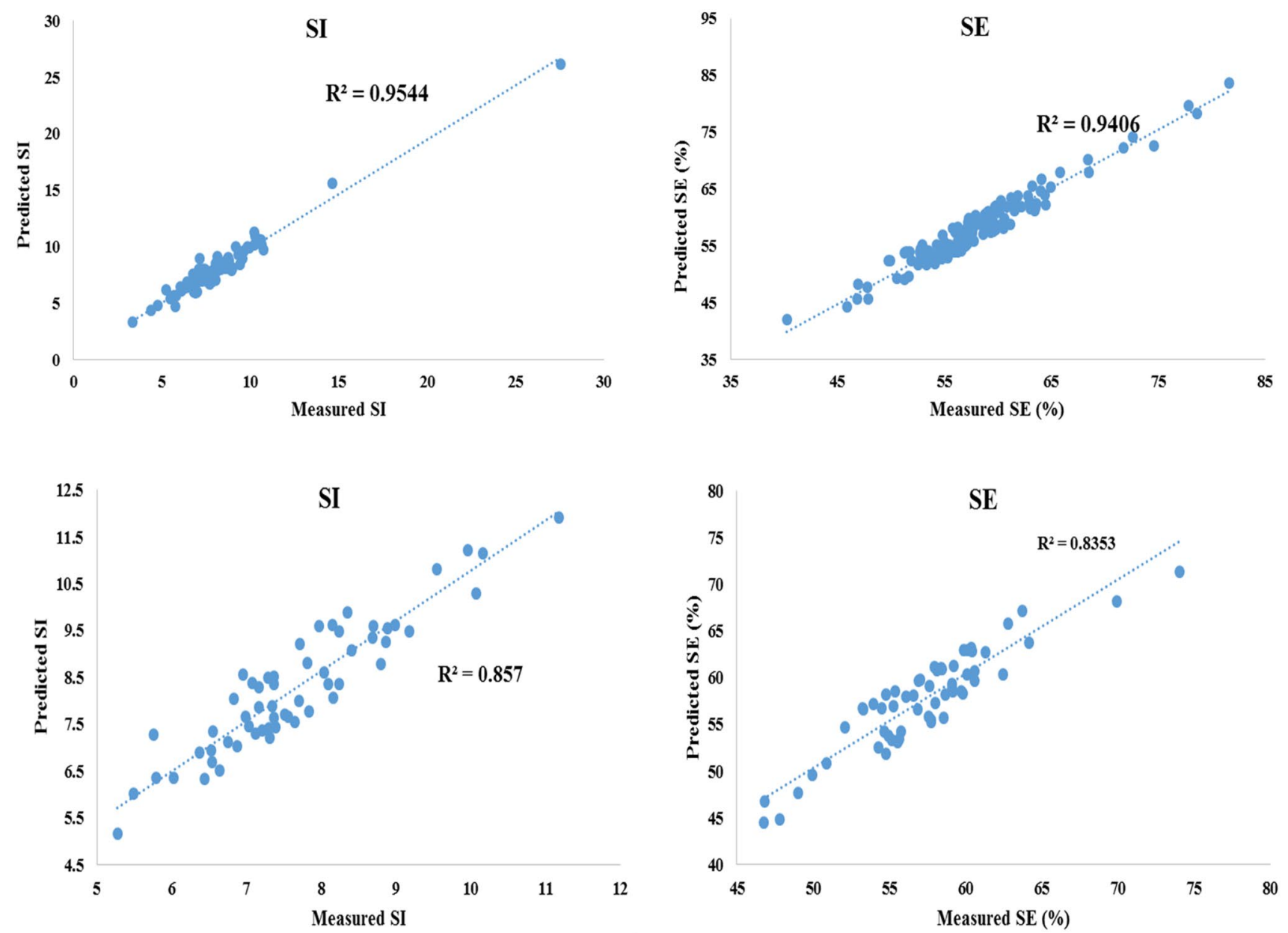

Fig. 3 Comparison of predicted and measured values for ANN model. First row: training stage; and second row: testing stage

Table 5 Comparison of statistical measures of ANN and ANN-GA in the training stage

Table 6 Comparison of statistical measures of ANN and ANN-GA in the testing stage

\begin{tabular}{|c|c|c|c|c|c|c|c|c|}
\hline \multirow[t]{2}{*}{ Method } & \multicolumn{4}{|c|}{ Selectivity index } & \multicolumn{4}{|c|}{ Separation efficiency } \\
\hline & $\mathrm{R}^{2}$ & MSE & MAD & MAPE & $\mathrm{R}^{2}$ & MSE & MAD & MAPE \\
\hline ANN & 0.95 & 0.220 & 0.266 & 3.297 & 0.94 & 2.372 & 1.340 & 2.342 \\
\hline ANN-GA & 0.99 & 0.052 & 0.166 & 2.222 & 0.99 & 0.043 & 0.152 & 0.264 \\
\hline
\end{tabular}

\begin{tabular}{|c|c|c|c|c|c|c|c|c|}
\hline \multirow[t]{2}{*}{ Method } & \multicolumn{4}{|c|}{ Selectivity index } & \multicolumn{4}{|c|}{ Separation efficiency } \\
\hline & $\mathrm{R}^{2}$ & MSE & MAD & MAPE & $\mathrm{R}^{2}$ & MSE & MAD & MAPE \\
\hline ANN & 0.86 & 0.635 & 0.629 & 8.277 & 0.84 & 4.646 & 1.889 & 3.310 \\
\hline ANN-GA & 0.95 & 0.276 & 0.294 & 3.542 & 0.92 & 1.782 & 1.097 & 1.947 \\
\hline
\end{tabular}

criteria calculated in the training and testing stages were relatively satisfactory that are presented in Tables 5 and 6 .

In GA-ANN model, the connection weights and biases of ANN are obtained by GA algorithm to avoid trapping in the local minima. As shown in Fig. 4, the value of fitness function (MSE) decreased after 100 generations. It is noted that the values of mean and best fitness are converged in which the best fitness for this model is 0.0438 . The chromosome (connection weights and biases) having the best fitness is saved for BP training.

After optimizing weights and biases for ANN, they are fed to the network for backpropagation training. The 
correlation coefficient for GA-ANN model in the training process is shown in Fig. 5 . Results indicated that the measured data and predicted data are close to each other in the training stage. After that, the test data are fed to the developed model for its validation. Figure 6

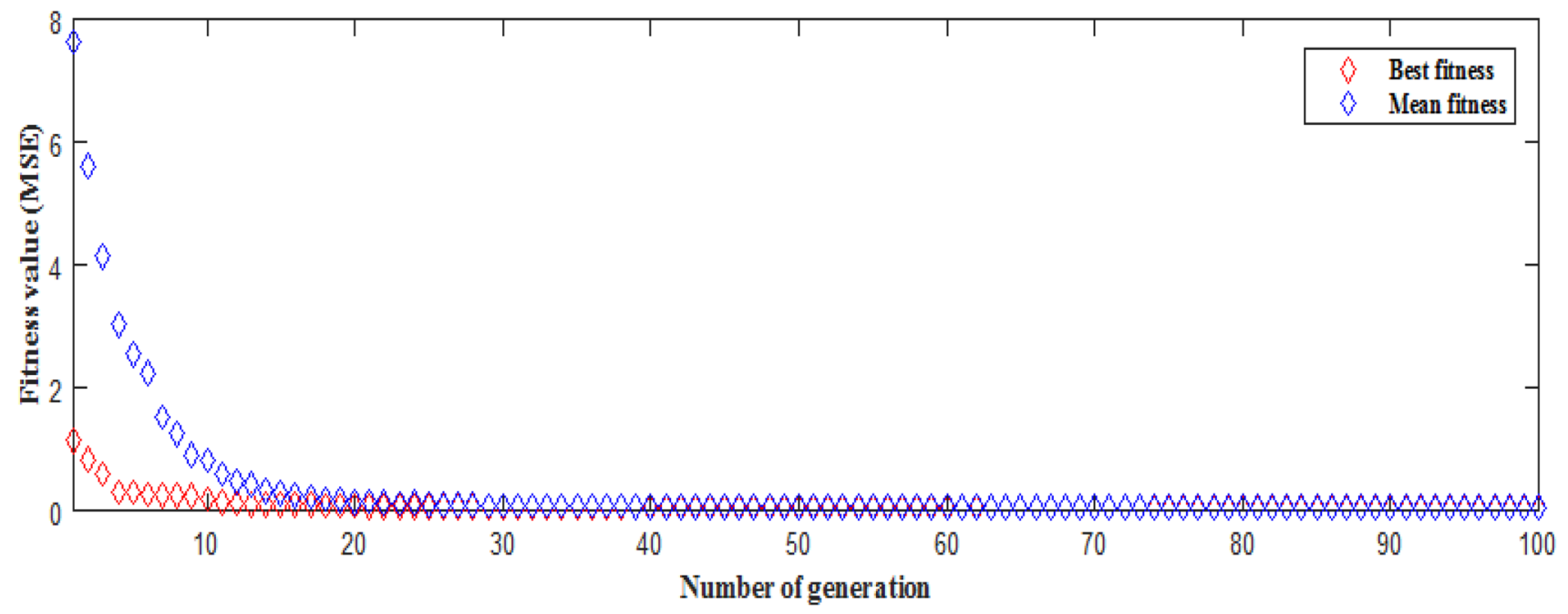

Fig. 4 Convergence of best fitness and mean fitness for this study
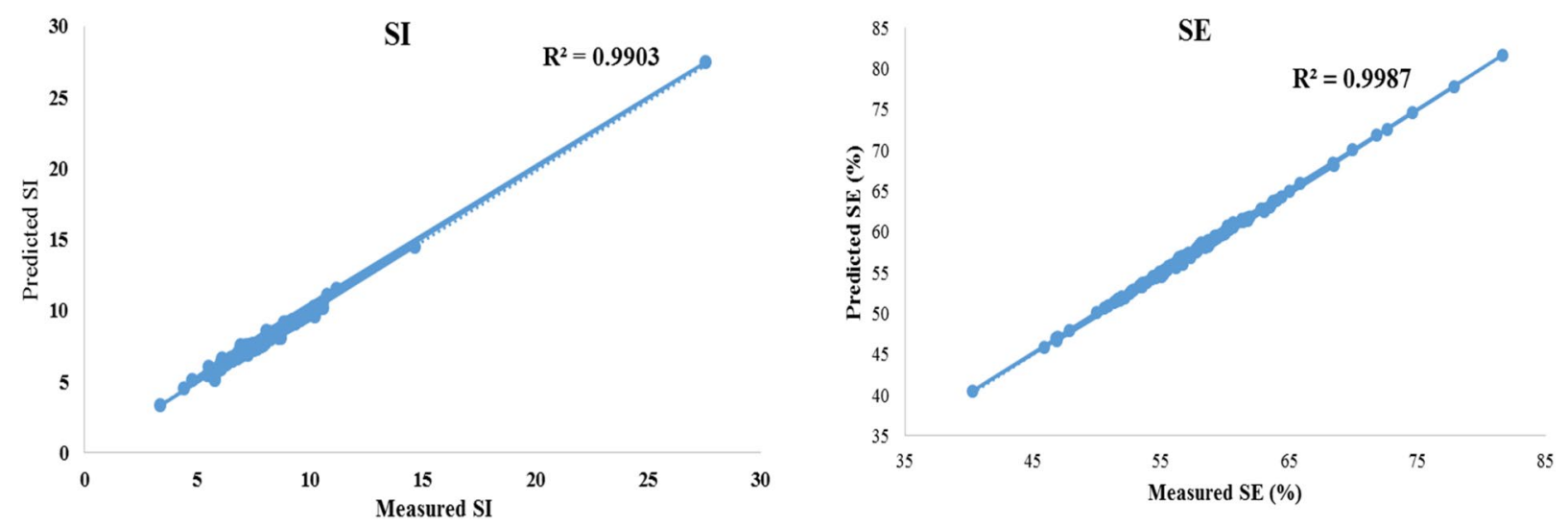

Fig. 5 Comparison of predicted and measured values for ANN-GA model in training stage
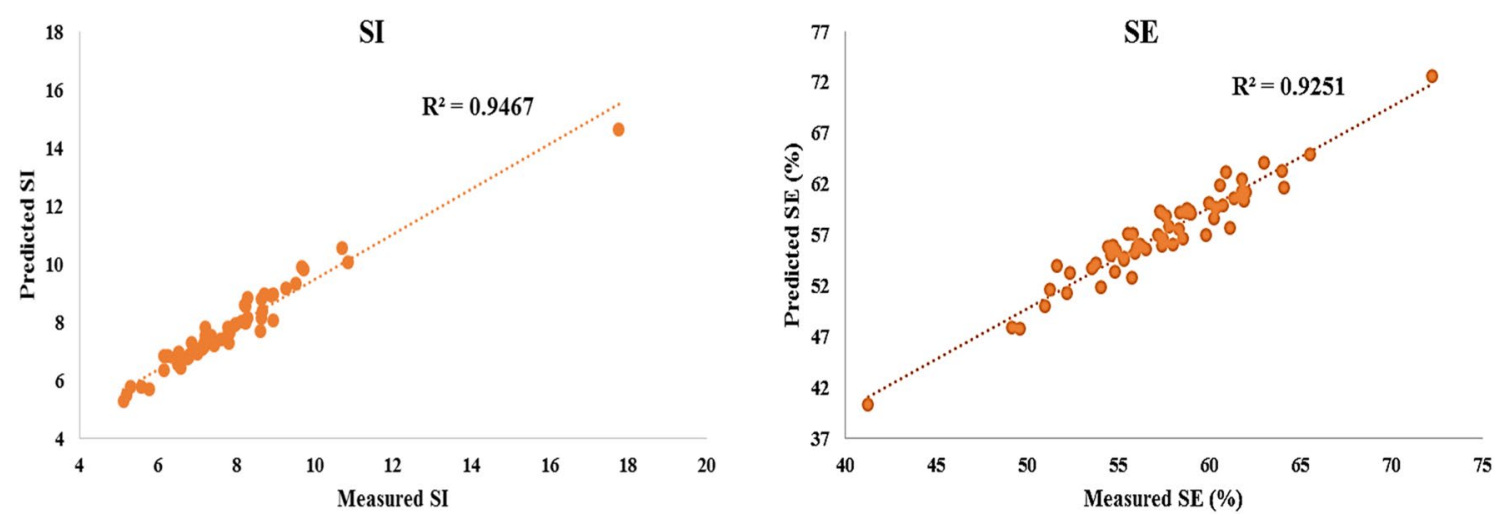

Fig. 6 Comparison of predicted and measured values for ANN-GA model in testing stage 
shows the comparison of predicted and measured values for ANN-GA model in testing stage.

The statistical criteria for evaluating the training and testing stages are given in Tables 5 and 6 . Results of modeling with ANN technique indicated that the quite mean square error and coefficient of determination for the testing phase were achieved 0.635 and 0.86 for selectivity index and of 4.646 and 0.84 for separation efficiency, respectively. To improve the performance of neural network, genetic algorithm was used to optimize the weights and biases of neural network. The results of modeling with the GA-ANN technique showed that the values of mean square error and coefficient of determination for the testing phase were obtained 0.276 and 0.95 for selectivity index and of 1.782 and 0.92 for separation efficiency, respectively. The other statistical criteria for the GA-ANN model were better than those of the ANN model. So, the GA-ANN efficiency for the prediction of the process outputs is more than ANN.

\section{Conclusions}

In this study, ANN and GA-ANN capabilities for predicting the separation efficiency and selectivity index in the industrial magnetic separation process were investigated. The affective separation parameters of hematite, including percentage of $\mathrm{Fe}, \mathrm{FeO}$ and $\mathrm{S}$ in mill feed and cobber feed, $80 \%$ passing size in mill feed and cobber feed and plant capacity were considered as input in the model development. The difference between the two models based on the statistical measures is tangible. The results indicated that the proposed model (GA-ANN) could be successfully applied to model the separation process of hematite under different operating conditions with a reasonable error. By optimizing the initial weights and biases, the ANN-GA model outperforms the ANN model significantly.

\section{Compliance with ethical standards}

Conflict of interest The authors declare that they have no conflict of interest.

Open Access This article is licensed under a Creative Commons Attribution 4.0 International License, which permits use, sharing, adaptation, distribution and reproduction in any medium or format, as long as you give appropriate credit to the original author(s) and the source, provide a link to the Creative Commons licence, and indicate if changes were made. The images or other third party material in this article are included in the article's Creative Commons licence, unless indicated otherwise in a credit line to the material. If material is not included in the article's Creative Commons licence and your intended use is not permitted by statutory regulation or exceeds the permitted use, you will need to obtain permission directly from the copyright holder. To view a copy of this licence, visit http://creativecommons .org/licenses/by/4.0/.

\section{References}

1. Liu Y, Sun F (2013) Parameter estimation of a pressure swing adsorption model for air separation using multi-objective optimisation and support vector regression model. Expert Syst Appl 40(11):4496-4502

2. Tohry A, Jafari M, Farahani M, Manthouri M, Chelgani SC (2020) Variable importance assessments of an innovative industrialscale magnetic separator for processing of iron ore tailings. Mineral Processing and Extractive Metallurgy. 65:1-8

3. Jahedsaravani A, Marhaban M, Massinaei M (2016) Application of statistical and intelligent techniques for modeling of metallurgical performance of a batch flotation process. Chem Eng Commun 203(2):151-160

4. Azqhandi MA, Ghaedi M, Yousefi F, Jamshidi M (2017) Application of random forest, radial basis function neural networks and central composite design for modeling and/or optimization of the ultrasonic assisted adsorption of brilliant green on ZnS-NP-AC. J Colloid Interface Sci 505:278-292

5. Allahkarami E, Nuri OS, Abdollahzadeh A, Rezai B, Chegini M (2016) Estimation of copper and molybdenum grades and recoveries in the industrial flotation plant using the artificial neural network. International Journal of Nonferrous Metallurgy 5(3):23-32

6. Allahkarami E, Igder A, Fazlavi A, Rezai B (2017) Prediction of $\mathrm{Co}$ (II) and Ni (II) ions removal from wastewater using artificial neural network and multiple regression models. Physicochemical Problems of Mineral Processing. 87:53

7. Allahkarami E, Salmani Nuri O, Abdollahzadeh A, Rezai B, Maghsoudi B (2017) Improving estimation accuracy of metallurgical performance of industrial flotation process by using hybrid genetic algorithm-artificial neural network (GA-ANN). Physicochemical Problems of Mineral Processing. 67:53

8. Anitha M, Singh H (2008) Artificial neural network simulation of rare earths solvent extraction equilibrium data. Desalination 232(1-3):59-70

9. Sepahvand P, Abdizadeh GR, Noori S (2020) Inverse design of an irregular-shaped radiant furnace using neural network and a modified hybrid optimization algorithm. Thermal Science and Engineering Progress 20:100730

10. Medi B, Bahramian A, Nazari V (2020) Synthesis and Characterization of Conducting Polyaniline Nanostructured Thin Films for Solar Cell Applications. JOM. 54:1-11

11. Tripathy SK, Mohanty I, Filippov LO (2020) Application of Artificial Neural Networks to Predict Dry Magnetic Separation of Low-Grade Hematite Fines. Transaction of the indian institute of metals. 73:1797-1807

12. Massinaei $M$, Sedaghati MR, Rezvani R, Mohammadzadeh AA (2014) Using data mining to assess and model the metallurgical efficiency of a copper concentrator. Chem Eng Commun 201(10):1314-1326

13. Hu W, Hadler K, Neethling SJ, Cilliers JJ (2013) Determining flotation circuit layout using genetic algorithms with pulp and froth models. Chem Eng Sci 102:32-41

14. Irannajad M, Nuri OS, Allahkarami E (2018) A new approach in separation process evaluation. Efficiency ratio and upgrading curves, Physicochemical Problems of Mineral Processing, p 54

15. Gaudin, A.M (1957) Flotation:McGraw-Hill 
16. Wills, B.A.; Finch, J (2015) Wills' mineral processing technology: an introduction to the practical aspects of ore treatment and mineral recovery:Butterworth-Heinemann

17. Salmani Nuri $O$, Allahkarami E, Irannajad M, Abdollahzadeh A (2017) Estimation of selectivity index and separation efficiency of copper flotation process using ANN model. Geosystem Engineering 20(1):41-50

18. Salmani Nuri O, Allahkarami E, Abdollahzadeh A (2017) Modeling and Optimization of SE and SI of Copper Flotation via Hybrid GA-ANN. Trans Indian Inst Met 70(9):2255-2263

19. Haykin, S (2007) Neural networks: a comprehensive foundation:Prentice-Hall, Inc.

20. Li C, Yang Z, Yan H, Wang $T$ (2014) The application and research of the GA-BP neural network algorithm in the MBR membrane fouling. Abstract and Applied Analysis, Hindawi. 2014:40-45

21. Davis, $L(1991)$ Handbook of genetic algorithms.

22. Sexton RS, Gupta JN (2000) Comparative evaluation of genetic algorithm and backpropagation for training neural networks. Inf Sci 129(1-4):45-59

23. Shopova EG, Vaklieva-Bancheva NG (2006) BASIC-A genetic algorithm for engineering problems solution. Comput Chem Eng 30(8):1293-1309
24. Yasin Y, Ahmad FBH, Ghaffari-Moghaddam M, Khajeh M (2014) Application of a hybrid artificial neural network-genetic algorithm approach to optimize the lead ions removal from aqueous solutions using intercalated tartrate-Mg-Al layered double hydroxides. Environmental Nanotechnology, Monitoring \& Management 1:2-7

25. Twomey J, Smith A (1995) Performance measures, consistency and power for artificial neural network models. Mathematical and computer modelling 21(1-2):243-258

26. Afkhami A, Abbasi-Tarighat M, Bahram M (2008) Artificial neural networks for determination of enantiomeric composition of a-phenylglycine using UV spectra of cyclodextrin host-guest complexes: Comparison of feed-forward and radial basis function networks. Talanta 75(1):91-98

Publisher's Note Springer Nature remains neutral with regard to jurisdictional claims in published maps and institutional affiliations. 